\title{
Optimal Recharge and Driving Strategies for a Battery-Powered Electric Vehicle
}

\author{
W.R. LEE, S. WANG and K.L. TEO* \\ School of Mathematics and Statistics, Curtin University of Technology, \\ GPO Box U 1987, Perth 6845, Australia
}

(Received 15 October 1998)

\begin{abstract}
A major problem facing battery-powered electric vehicles is in their batteries: weight and charge capacity. Thus, a battery-powered electric vehicle only has a short driving range. To travel for a longer distance, the batteries are required to be recharged frequently. In this paper, we construct a model for a battery-powered electric vehicle, in which driving strategy is to be obtained such that the total travelling time between two locations is minimized. The problem is formulated as an optimization problem with switching times and speed as decision variables. This is an unconventional optimization problem. However, by using the control parametrization enhancing technique (CPET), it is shown that this unconventional optimization is equivalent to a conventional optimal parameter selection problem. Numerical examples are solved using the proposed method.
\end{abstract}

Keywords: Optimal control; Electric vehicle; Optimal battery recharge

\section{INTRODUCTION}

Due to the advancement in the battery technology, there is a great revival of interest among the researchers to return to work on the development of battery-powered electric vehicles over the past decade. For details, see $[1,6-8]$.

\footnotetext{
* Corresponding author. Tel.: 61892663483 . Fax: 61892663197.

E-mail: teo@cs.curtin.edu.au.
} 
The main components of an electric vehicle are: (i) battery cells which are energy storage system; (ii) a traction system which consists of an electric motor, a steeling wheel and a motor controller; and (iii) a friction system which is a mechanical braking device. The driver can control the power applied to the traction system as well as to the mechanical braking system. The major problem associated with a battery-powered electric vehicle is in its batteries. The total weight of the batteries is directly related to the charging capacity of the electric vehicle. Due to the limited charging capacity, the vehicle can only travel for a relatively short distance when compared with a conventional gasoline-powered vehicle. It needs to be recharged much more frequently.

In this paper, we consider a situation in which an electric vehicle is to be driven on an even road or an undulated road connecting two given cities. The distance between the two cities is beyond the driving range of the vehicle without recharge. In other words, the batteries are required recharging before completing the journal. The rest of the paper is organized as follows:

We construct a model for the battery-powered electric vehicle in Section 2. In Section 3, we formulate an optimization problem in which the vehicle is to be driven on an even road. The recharge points, and the speed of the vehicle are considered as decision variables. The objective is to minimize the completion time of the journey with respect to these decision variables. The distance between the two cities, the maximum allowable power to be applied to the motor, and the number of battery cells are assumed fixed. In this optimization problem, the capacity of each of the battery cells is regarded as the state. Clearly, the state will exhibit a jump at each switching time. We further assume that the maximum allowable number of recharge points is fixed. A challenging task is to remove this assumption in that theoretical results are obtained for determining this maximum number of recharge points. To solve this optimization problem, we first fix a number of recharge points. This leads to simpler problem in which only the switching times and the vehicle speed are to be chosen optimally. The control parametrization enhancing transform (CPET) (see [4] for details) is then used to transform the problem into a standard optimal control problem solving by MISER3.2 (cf. [2,3]). We then increase the number of the recharge points and solve the 
corresponding standard optimal control problem. This process is repeated by gradually increasing the number of the recharge points until the maximum allowable number is reached. In Section 4, we formulate an optimization problem in which the recharge points are to be chosen from several fixed points rather than free as in Section 3. A computational method similar to that given in Section 3 is developed for solving this optimization problem. Note that in both Sections 3 and 4 the vehicle is driven on an even road. In Sections 5 and 6, we consider the case in which the vehicle is driven on an undulated road. In Section 5, we formulate an optimization problem in which the recharge points are fixed, and the switching times and the corresponding speed of the vehicle are considered as decision variables. In Section 6 , recharge points as well as the switching times and the corresponding speed of the vehicle are taken as decision variables. In Section 7, four examples are constructed and solved by using the methods developed in the previous sections.

\section{BATTERY-POWERED ELECTRIC CAR MODEL}

Let $t$ denote the time, and $p$ the power being applied to the motor. The power $p$, which is a function of $t$, is a control variable. It is assumed that

$$
0 \leq p(t) \leq P, \quad \text { for all } t \in\left[0, t_{\text {final }}\right]
$$

where $t_{\text {final }}$ is the specified time for completing the journey, and $P$ is the maximum allowable power to be applied to the motor. The electric power is directly applied to the motor. We assume that the control force at the wheel of the vehicle is:

$$
F=\frac{p}{v}
$$

where $v=v(t)$ is the speed of the vehicle. We also assume that the resistance on the vehicle due to the friction and air is:

$$
r(v)=m\left(b_{1}+b_{2} v\right)
$$

where $b_{1}$ and $b_{2}$ are known positive constants, and $m$ is the mass of the vehicle. The dynamics of the vehicle is described by the following 
system of differential equations:

$$
\begin{aligned}
\frac{\mathrm{d} x}{\mathrm{~d} t} & =v, \\
m \frac{\mathrm{d} v}{\mathrm{~d} t} & =\frac{p}{v}-r(v)-m g \sin (\theta)
\end{aligned}
$$

with initial conditions

$$
\begin{aligned}
& v(0)=0, \\
& x(0)=0,
\end{aligned}
$$

where $\theta=\theta(x)$ is the angle of slope of the road at location $x=x(t)$, and $g$ is the acceleration due to gravity.

The speed of the vehicle is assumed to satisfy the following obvious constraint.

$$
v(t) \geq 0
$$

For each battery cell, the recharge-discharge rate at time $t$ is:

$$
\frac{\mathrm{d} q}{\mathrm{~d} t}= \begin{cases}-c_{1} p_{1}, & \text { discharge } \\ \left(c_{2} /\left(q+c_{3}\right)\right)-\exp \left\{c_{4}-c_{5} t\right\}, & \text { recharge }\end{cases}
$$

with initial condition:

$$
q(0)=q_{0},
$$

where $c_{i}, i=1, \ldots, 5$, are given positive constants, and $p_{1}$ is the power flowing out from each battery cell. The function $q$ is required to satisfy the following constraint:

$$
q_{\min } \leq q(t) \leq q_{\max } .
$$

\section{THE PROBLEM ON AN EVEN ROAD}

In this section, the road connecting two given cities is assumed to be even. The distance between the two cities, the maximum allowable power to be applied to the motor, and the number of battery cells are assumed to be fixed. 


\subsection{Problem Formulation}

Let $\left[0, t_{\text {final }}\right]$ be the total driving time, and let $\left\{t_{i}\right\}, i=0,1, \ldots, 2 l$, be switching times, which satisfy

$$
0=t_{0}<t_{1}<\cdots<t_{2 l}<t_{2 l+1}=t_{\text {final }},
$$

where $l$ is the number of recharge points, $\left[t_{2 i-1}, t_{2 i}\right], i=1, \ldots, l$, are the time intervals at which the vehicle stops for recharging its batteries, while $\left[t_{2 i-2}, t_{2 i-1}\right], i=1, \ldots, l+1$, are the time intervals at which the vehicle is driven at a constant speed. We ignore the times taken for acceleration (respectively, deceleration) at the beginning (respectively, ending) of each of the time intervals. Thus, in each time interval, we obtain

$$
\frac{\mathrm{d} v}{\mathrm{~d} t}=0
$$

From (2.2), (2.4), and (3.1), we obtain

$$
\begin{aligned}
p & =v \times r(v) \\
& =v \times m\left(b_{1}+b_{2} v\right)
\end{aligned}
$$

and the power flowing out of each of the $n$ parallelly connected battery cells is:

$$
p_{1}=v \times \frac{m\left(b_{1}+b_{2} v\right)}{n} .
$$

From (2.7) and (3.3), we have

$$
\frac{\mathrm{d} q}{\mathrm{~d} t}= \begin{cases}-c_{1} \frac{\left(b_{1} v+b_{2} v^{2}\right) \cdot m}{n}, & t \in\left[t_{2 i-2}, t_{2 i-1}\right), \\ \frac{c_{2}}{q+c_{3}}-\exp \left\{c_{4}-c_{5}\left(t-t_{2 i-1}\right)\right\}, & t \in\left[t_{2 i-1}, t_{2 i}\right), \\ -c_{1} \frac{\left(b_{1} v+b_{2} v^{2}\right) \cdot m}{n}, & t \in\left[t_{2 l}, t_{2 l+1}\right], \\ i=1, \ldots, l & \end{cases}
$$

with initial condition

$$
q(0)=q_{0}
$$


Define

$$
\mathcal{T}=\left\{\boldsymbol{t}=\left(t_{1}, \ldots, t_{2 l}\right): t_{i} \in \mathbb{R}, t_{i-1}<t_{i}, i=1, \ldots, 2 l, l \in \mathbb{N}\right\},
$$

where $\mathbb{R}$ and $\mathbb{N}$ are set of all real numbers and set of all natural numbers, respectively. A measurable function $v:[0, T] \rightarrow \mathbb{R}$ is called an admissible control. Let $\mathcal{V}$ be the set of all such admissible controls. For each $(\boldsymbol{t}, v, l) \in \mathcal{T} \times \mathcal{V} \times \mathbb{N}$, let $q(\cdot \mid \boldsymbol{t}, v, l)$ denote the corresponding solution of system (3.4) and (3.5). We may now specify the corresponding optimization problem formally as follows:

Given the dynamical system (3.4) and (3.5), find a $(t, v, l) \in$ $\mathcal{T} \times \mathcal{V} \times \mathbb{N}$ such that the cost function

$$
g_{1}(\boldsymbol{t}, v, l)=t_{\text {final }}(\boldsymbol{t}, v, l)
$$

is minimized subject to the constraints

$$
\begin{aligned}
& q_{\min } \leq q(t) \leq q_{\max } \\
& 0<p=m\left(a v+b v^{2}\right)<P \\
& 0<t_{i}-t_{i-1}, \quad i=1, \ldots, 2 l+1, \\
& \int_{0}^{t_{\text {final }}} v \mathrm{~d} t=S, \\
& l \leq L
\end{aligned}
$$

where $n, l$ and $v$ are, respectively, the number of battery cells, number of recharge points, and the corresponding speed. $t_{\text {final }}=t_{2 l+1}$ is the required time for completing the journey, $S$ is the total distance travelled, and $L$ is the upper bound of the number of the recharge points. Let this problem be referred to as Problem $P_{1}$.

\subsection{Transformation}

To solve Problem $P_{1}$, we need to determine the optimal number of recharge points $l$, the optimal switching time points $t=\left(t_{1}, \ldots, t_{2 l}\right)$, and the optimal speed $v$. Let us initially set the number of recharge points to be $k$. Then only the optimal switching time points with a fixed number of recharge points, and the corresponding optimal speed are to be determined. This simplified problem is referred to as Problem $P_{1}^{k}$. 
We now apply the CPET $[4,5]$ to Problem $P_{1}^{k}$. Let $s \in[0,2 k+1]$ be a new time variable, and define

$$
\eta(s)=\sum_{i=1}^{2 k+1} \eta_{i} \chi_{[i-1, i)}(s),
$$

where $\chi_{[i-1, i)}(s)$ is the characteristic function on the interval $[i-1, i)$, and the $\eta_{i}^{\prime} s$ are nonnegative constants. Clearly, $\eta(s)$, which is called an enhancing control, is a nonnegative piecewise constant function defined on $[0,2 k+1]$ with fixed switching points $\{1,2, \ldots, 2 k\}$.

The CPET:

$$
\frac{\mathrm{d} t}{\mathrm{~d} s}=\eta(s), \quad t(0)=0
$$

maps $t \in\left[0, t_{\text {final }}\right]$ to $s \in[0,2 k+1]$, where

$$
\eta(s)=t_{i}-t_{i-1}, \quad s \in[i-1, i), \quad i=1,2, \ldots, 2 k+1
$$

satisfying

$$
\int_{0}^{2 k+1} \eta(s) \mathrm{d} s=t_{\text {final }} .
$$

Let $\Theta$ denote the class of all such enhancing controls. Under the CPET, the system dynamics (3.4) and (3.5) becomes

$$
\begin{aligned}
& \frac{\mathrm{d}}{\mathrm{d} s}\left(\begin{array}{c}
\hat{q}(s) \\
t(s)
\end{array}\right)
\end{aligned}
$$

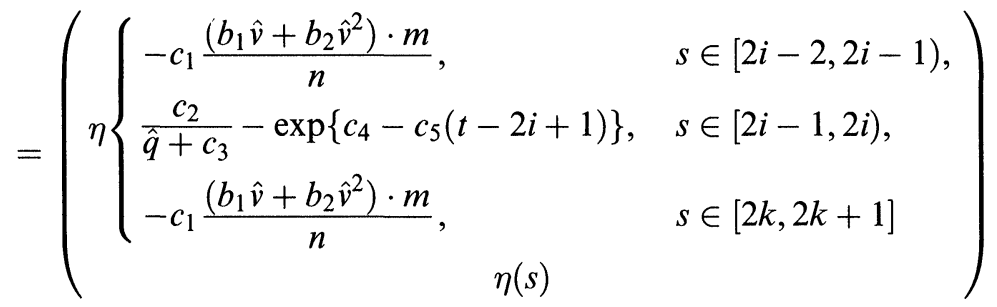

$i=1, \ldots, l$ with initial condition

$$
\left(\begin{array}{c}
\hat{q}(0) \\
t(0)
\end{array}\right)=\left(\begin{array}{c}
q_{0} \\
0
\end{array}\right)
$$


where $\hat{q}(s)=q(t(s)), \hat{v}(s)=v(t(s)), \quad s \in[0,2 k+1]$ and $i=1, \ldots, k$, while the constraints (3.7) $-(3.11)$ reduce to

$$
\begin{aligned}
& q_{\min } \leq \hat{q}(s) \leq q_{\max }, \\
& 0<p=m\left(b_{1} \hat{v}+b_{2} \hat{v}^{2}\right)<P, \\
& 0<\eta_{i}, \quad i=1, \ldots, 2 k+1, \\
& \int_{0}^{2 k+1} \hat{v} \mathrm{~d} s=S, \\
& l \leq L .
\end{aligned}
$$

LEMMA 3.1 The all-time state constraint (3.14) is equivalent to the following terminal state inequality constraints:

$$
\begin{aligned}
q_{\min } & \leq \hat{q}(2 i-1), & & i=1, \ldots, k+1, \\
\hat{q}(2 i) & \leq q_{\max }, & & i=1, \ldots, k .
\end{aligned}
$$

Proof Obviously: (3.14) $\Longrightarrow(3.19)$ and (3.20). Conversely, suppose that (3.19) and (3.20) exist. Then, for each $i=1, \ldots, k+1, \hat{q}(s)$, $s \in[2 i-2,2 i-1]$, is monotonically decreasing, since the vehicle is driven at a constant speed and no battery recharge is taken place in this sub-interval. Thus

$$
q_{\min } \leq \hat{q}(2 i-1) \leq \hat{q}(s) \leq \hat{q}(2 i-2) \leq q_{\max } .
$$

For each $i=1, \ldots, k, \hat{q}(s), s \in[2 i-1,2 i]$, is monotonically increasing, since the vehicle is stopped for recharging the batteries in this subinterval. Thus

$$
q_{\min } \leq \hat{q}(2 i-1) \leq \hat{q}(s) \leq \hat{q}(2 i) \leq q_{\max } .
$$

Therefore, the condition (3.14) exists, and the result follows.

Let $\mathcal{V}_{1}$ be the set of all those feasible $\hat{v}(s)$, such that the conditions (3.15)-(3.20) are satisfied. Problem $P_{1}^{k}$ is now transformed into the following optimal control problem: Given the dynamical system (3.12) 
and (3.13), find a $(\boldsymbol{\eta}, \hat{v}) \in \Theta \times \mathcal{V}_{1}$ such that the cost function

$$
\hat{g}_{1}(\eta, \hat{v})=\sum_{i=1}^{2 k+1} \eta_{i}
$$

is minimized subject to the constraints (3.15)-(3.20).

This problem is referred to as Problem $P_{1}^{k 0}$.

Definition $3.1 \quad(\boldsymbol{\eta}, \hat{v}) \in \Theta \times \mathcal{V}_{1}$ (respectively, $\left.(\boldsymbol{t}, \boldsymbol{v})\right)$ is said to be a feasible element if the constraints (3.15)-(3.20) (respectively, constraints (3.7)-(3.11)) are satisfied.

Theorem 3.2 Problem $\left(P_{1}^{k 0}\right)$ is equivalent to Problem $\left(P_{1}^{k}\right)$ in the sense that $\left(\boldsymbol{\eta}^{*}, \hat{v}^{*}\right)$, is a solution of Problem $\left(P_{1}^{k 0}\right)$ if and only if $\left(\boldsymbol{t}^{*}, v^{*}\right)$ is a solution of problem $\left(P_{1}^{k}\right)$, and $\hat{g}_{1}\left(\boldsymbol{\eta}^{*}, \hat{v}^{*}\right)=g_{1}\left(\boldsymbol{t}^{*}, v^{*}\right)$, where $\boldsymbol{\eta}^{*}(\boldsymbol{s})=$ $t_{i}^{*}-t_{i-1}^{*}, \quad s \in[i-1, i], \quad i=1,2, \ldots, 2 k+1$, and $\hat{v}^{*}(s)=v\left(t^{*}(s)\right), \quad s \in[0$, $2 k+1]$.

Proof Let $\left(\boldsymbol{t}^{1}, v^{1}\right) \in \mathcal{T} \times \mathcal{V}$ be a feasible element of Problem $\left(P_{1}^{k}\right)$, and let $(\boldsymbol{\eta}, \hat{v}) \in \Theta \times \mathcal{V}_{1}$ be the corresponding feasible element of Problem $\left(P_{1}^{k 0}\right)$. Then it is easy to check that $q(t)$ is the solution of (3.4) and (3.5) (with $l=k$ ) if and only if $\hat{q}(s)$ is the solution of (3.12) and (3.13), and

$$
g_{1}(\boldsymbol{t}, v)=t_{\text {final }}(\boldsymbol{t}, v)=\sum_{i=1}^{2 k+1}\left(t_{i}-t_{i-1}\right)=\sum_{i=1}^{2 k+1} \eta_{i}=\hat{g}_{1}(\boldsymbol{\eta}, \hat{v}) .
$$

Hence, the result follows readily.

In Problem $\left(P_{1}^{k}\right)$ the cost function (3.6) is minimized with respect to $(\boldsymbol{t}, v) \in \mathcal{T} \times \mathcal{V}$ where $\boldsymbol{t}=\left(t_{1}, t_{2}, \ldots, t_{2 k}\right)$ is the vector of switching time points. On the other hand, the cost function (3.21) in Problem $\left(P_{1}^{k 0}\right)$ is minimized with respect to $(\boldsymbol{\eta}, \hat{v}) \in \Theta \times \mathcal{V}_{1}$ where $\boldsymbol{\eta}(t)$ is a nonnegative piecewise constant function. Problem $\left(P_{1}^{k 0}\right)$ is equivalent to Problem $\left(P_{1}^{k}\right)$. However, Problem $\left(P_{1}^{k 0}\right)$ is numerically much more tractable, because it does not involve variable switching times.

Let us now address the question of finding the optimal number $l$ of the recharge points. Let $\boldsymbol{t}=\left(t_{1}, \ldots, t_{2 k}\right)$ be optimal solution of Problem $\left(P_{1}^{k}\right)$ corresponding to the positive integer $k$. We propose the following algorithm. 


\section{Algorithm A}

1. Choose an initial guess $k=k_{0}$.

2. Let $t_{0}=0, t_{2 k+1}=t_{\text {final }}$, and solve Problem $\left(P_{1}^{k}\right)$ to obtain $\boldsymbol{t}=\left(t_{1}, \ldots, t_{2 k}\right)$. Let $\hat{g}_{1}^{k}$ be the corresponding optimal cost.

3. If $k=L$, stop, find $k^{*}$ such that $\hat{g}_{1}^{k^{*}} \leq \hat{g}_{1}^{l}$ for all $l \in\left\{k_{0}, \ldots, L\right\}$, and $k^{*}$ is the optimal number of the recharge points. Otherwise, let $k=k+1$ and go to step 2 .

\section{FIXED RECHARGE LOCATIONS}

Following the notation defined before and again consider the situation in which the road is even. However, we assume that the vehicle can only be recharged at a given set of locations $\boldsymbol{\tau}=\left\{\tau_{1}, \ldots, \tau_{h}\right\}$, satisfying $0<\tau_{1}<\tau_{2}<\cdots<\tau_{h}<S$, where $S$ is the maximum distance the vehicle is expected to travel as defined in the previous section. We assume that $h$ is fixed. We also assume that the number of recharge points $l=h$. The problem can then be formulated as the following optimization problem:

Given the dynamical system (3.4) and (3.5) with $l=h$, find a $(\boldsymbol{t}, v) \in \mathcal{T} \times \mathcal{V}$ such that the cost function

$$
g_{2}(\boldsymbol{t}, v)=t_{\text {final }}(\boldsymbol{t}, v)
$$

is minimized subject to the constraints

$$
\begin{aligned}
& q_{\min } \leq q(t) \leq q_{\max }, \\
& 0<p=m\left(a v+b v^{2}\right)<P, \\
& 0<t_{i}-t_{i-1}, \quad i=1, \ldots, 2 h+1, \\
& \int_{0}^{t_{\text {final }}} v \mathrm{~d} t=S, \\
& \int_{0}^{t_{2 i-1}} v(t) \mathrm{d} t=\tau_{i}, \quad i=1, \ldots, h .
\end{aligned}
$$

This problem is referred to as Problem $P_{2}$.

To begin, we choose the number of recharge points to be $h$. However, the exact optimal number of recharge points is equal to $h$ 
minus the number of those recharge points corresponding to the occurrance of redundancies.

Under the CPET, the constraints (4.2)-(4.6) reduce to

$$
\begin{aligned}
& q_{\min } \leq \hat{q}(2 i-1), \quad i=1, \ldots, h+1, \\
& \hat{q}(2 i) \leq q_{\max }, \quad i=1, \ldots, h, \\
& 0<p=m\left(b_{1} \hat{v}+b_{2} \hat{v}^{2}\right)<P, \\
& 0<\eta_{i}, \quad i=1, \ldots, 2 h+1, \\
& \int_{0}^{2 h+1} \hat{v} \mathrm{~d} s=S, \\
& \int_{0}^{2 i-1} v(s) \mathrm{d} s=\tau_{i}, \quad i=0, \ldots, h .
\end{aligned}
$$

Let $\mathcal{V}_{2}$ be the set of all those $\hat{v}(s)$, such that the conditions (4.7)(4.12) are satisfied. Problem $P_{2}$ is now transformed into the following optimal control problem.

Given the dynamical system (3.12) and (3.13) (with $k=h$ ), find an admissible element $(\boldsymbol{\eta}, \hat{v}) \in \Theta \times \mathcal{V}_{2}$ such that the cost function

$$
\hat{g}_{2}(\boldsymbol{\eta}, \hat{v})=\sum_{i=1}^{2 h+1} \eta_{i}
$$

is minimized subject to the constraints (4.7)-(4.12).

This problem is referred to as Problem $P_{2}^{0}$.

Theorem 4.1 Problem $\left(P_{2}^{0}\right)$ is equivalent to Problem $\left(P_{2}\right)$ in the sense that $\left(\boldsymbol{\eta}^{*}, \hat{v}^{*}\right)$ is a solution of Problem $\left(P_{2}^{0}\right)$ if and only if $\left(\boldsymbol{t}^{*}, v^{*}\right)$ is a solution of Problem $\left(P_{2}\right)$, and $\hat{g}_{2}\left(\eta^{*}, \hat{v}^{*}\right)=g_{2}\left(t^{*}, v^{*}\right)$, where $\eta^{*}(s)=$ $t_{i}^{*}-t_{i-1}^{*}, \quad s \in[i-1, i), \quad i=1,2, \ldots, 2 h+1, \quad$ and $\hat{v}^{*}(s)=v\left(t^{*}(s)\right)$, $s \in[0,2 h+1]$.

Proof The proof is similar to the proof for Theorem 3.2. 


\section{UNDULATED ROAD}

In this section, the road connecting the two cities is assumed to be undulated. The distance between the two cities, the maximum allowable power to be applied to the motor, and the number of battery cells are again assumed to be fixed. $\boldsymbol{\tau}=\left\{\tau_{1}, \ldots, \tau_{l}\right\}$, satisfying $0<\tau_{1}<$ $\tau_{2}<\cdots<\tau_{l}<S$, are recharge locations at which the vehicle can be recharged. These recharge locations indude the turning points $\boldsymbol{\gamma}=\left(\gamma_{1}, \ldots, \gamma_{m}\right)$ of undulated road. The number of recharge points, $l$, is assumed to be fixed.

\subsection{Problem Formulation}

Since the road connecting the two cities is undulated, the power being applied to the motor (see (3.2)) becomes

$$
\begin{aligned}
p & =v \times r(v) \\
& =v \times m\left(b_{1}+b_{2} v+g \sin \theta\right),
\end{aligned}
$$

and the power flowing out of each of the $n$ parallelly connected battery cells (see (3.3)) becomes

$$
p_{1}=v \times \frac{m\left(b_{1}+b_{2} v+g \sin \theta\right)}{n} .
$$

From (2.7) and (5.1), we have

$$
\frac{\mathrm{d} q}{\mathrm{~d} t}= \begin{cases}-c_{1} \frac{\left(b_{1} v+b_{2} v^{2}+g \sin \theta\right) \cdot m}{n}, & t \in\left[t_{2 i-2}, t_{2 i-1}\right), \\ \frac{c_{2}}{q+c_{3}}-\exp \left\{c_{4}-c_{5}\left(t-t_{2 i-1}\right)\right\}, & t \in\left[t_{2 i-1}, t_{2 i}\right), \\ -c_{1} \frac{\left(b_{1} v+b_{2} v^{2}+g \sin \theta\right) \cdot m}{n}, & t \in\left[t_{2 l}, t_{2 l+1}\right], \\ i=1, \ldots, l & \end{cases}
$$

with initial condition

$$
q(0)=q_{0}
$$


We now define the corresponding optimization problem formally as follows:

Find a $(t, v) \in \mathcal{T} \times \mathcal{V}$ such that the cost function

$$
g_{3}(\boldsymbol{t}, v)=t_{\text {final }}(\boldsymbol{t}, v)
$$

is minimized subject to the system (5.2) and (5.3) and the following constraints:

$$
\begin{aligned}
& q_{\min } \leq q(t) \leq q_{\max }, \\
& 0<p=m\left(a v+b v^{2}+g \sin \theta\right)<P, \\
& 0<t_{i}-t_{i-1}, \quad i=1, \ldots, 2 l+1, \\
& \int_{0}^{t_{\text {final }}} v \mathrm{~d} t=S, \\
& \int_{0}^{t_{2 i-1}} v(t) \mathrm{d} t=\tau_{i}, \quad i=1, \ldots, l,
\end{aligned}
$$

where $S$ is the total distance travelled, $n$ and $l$ are, respectively, the number of battery cells and number of recharge times, $v$ is the speed, and $\boldsymbol{\tau}=\left\{\tau_{1}, \ldots, \tau_{l}\right\}$ is the vector of recharge points. Let this problem be referred to as Problem $P_{3}$.

\subsection{Transformation}

To find optimal solution of Problem $P_{3}$, we need to determine the optimal time points $t=\left(t_{1}, \ldots, t_{2 l}\right)$, and the optimal speed $v$.

We now apply the CPET [4,5] to Problem $P_{3}$. Let $s \in[0,2 l+1]$ be a new time variable, and let $\eta(s)$ be defined by

$$
\eta(s)=\sum_{i=1}^{2 l+1} \eta_{i} \chi_{[i-1, i)}(s),
$$

where $\chi_{[i-1, i)}(s)$ is the characteristic function on the interval $[i-1, i)$, and the $\eta_{i}^{\prime} s$ are nonnegative constants. Clearly, $\eta(s)$, which is called the enhancing control, is a nonnegative piecewise constant function defined on $[0,2 l+1]$ with fixed switching points $\{1,2, \ldots, 2 l\}$. The CPET

$$
\frac{\mathrm{d} t}{\mathrm{~d} s}=\eta(s), \quad t(0)=0
$$


maps $t \in\left[0, t_{\text {final }}\right]$ into $s \in[0,2 l+1]$, where

$$
\eta(s)=t_{i}-t_{i-1}, \quad s \in[i-1, i), \quad i=1,2, \ldots, 2 l+1,
$$

satisfying

$$
\int_{0}^{2 l+1} \eta(s) \mathrm{d} s=t_{\text {final }} .
$$

Let $\Theta$ denote the class of all such enhancing controls. Under the CPET, the system dynamics (5.2) and (5.3) becomes

$$
\begin{aligned}
& \frac{\mathrm{d}}{\mathrm{d} s}\left(\begin{array}{c}
\hat{q}(s) \\
t(s)
\end{array}\right) \\
& =\left(\begin{array}{ll}
\left(\begin{array}{cc}
-c_{1} \frac{\left(b_{1} \hat{v}+b_{2} \hat{v}^{2}+g \sin \theta\right) \cdot m}{n}, & s \in[2 i-2,2 i-1), \\
\frac{c_{2}}{\hat{q}+c_{3}}-\exp \left\{c_{4}-c_{5}(t-2 i+1)\right\}, & s \in[2 i-1,2 i), \\
-c_{1} \frac{\left(b_{1} \hat{v}+b_{2} \hat{v}^{2}+g \sin \theta\right) \cdot m}{n}, & s \in[2 l, 2 l+1] \\
\eta(s) &
\end{array}\right)
\end{array}\right.
\end{aligned}
$$

$i=1, \ldots, l$ with the initial condition

$$
\left(\begin{array}{c}
\hat{q}(0) \\
t(0)
\end{array}\right)=\left(\begin{array}{c}
q_{0} \\
0
\end{array}\right)
$$

where $\hat{q}(s)=q(t(s)), \hat{v}(s)=v(t(s)), s \in[0,2 l+1]$, and $i=1, \ldots, l$.

The constraints (5.5) $-(5.9)$ reduce to

$$
\begin{array}{ll}
q_{\min } \leq \hat{q}(2 i-1), & i=1, \ldots, l+1, \\
\hat{q}(2 i) \leq q_{\max }, & i=1, \ldots, l, \\
0<p=m\left(b_{1} \hat{v}+b_{2} \hat{v}^{2}+g \sin \theta\right)<P, & i=1, \ldots, 2 l+1, \\
0<\eta_{i}, & \\
\int_{0}^{2 l+1} \hat{v} \mathrm{~d} s=S, & \\
\int_{0}^{2 i-1} v(s) \mathrm{d} s=\tau_{i}, & i=0, \ldots, l .
\end{array}
$$


Let $\mathcal{V}_{3}$ be the set of all those $\hat{v}(s)$, such that the conditions (5.12)(5.17) are satisfied. Problem $P_{3}$ is now transformed into the following optimal control problem:

Given the dynamical system (5.10)-(5.11), find a $(\boldsymbol{\eta}, \hat{v}) \in \Theta \times \mathcal{V}_{3}$ such that the cost function

$$
\hat{g}_{3}(\boldsymbol{\eta}, \hat{v})=\sum_{i=1}^{k+1} \eta_{i}
$$

is minimized subject to the constraints (5.12)-(5.17).

This problem is referred to as Problem $P_{3}^{0}$.

Theorem 5.1 Problem $\left(P_{3}^{0}\right)$ is equivalent to Problem $\left(P_{3}\right)$ in the sense that $\left(\boldsymbol{\eta}^{*}, \hat{v}^{*}\right)$ is a solution of Problem $\left(P_{3}^{0}\right)$ if and only if $\left(\boldsymbol{t}^{*}, v^{*}\right)$ is a solution of Problem $\left(P_{3}\right)$, and $\hat{g}_{3}\left(\eta^{*}, \hat{v}^{*}\right)=g_{3}\left(\boldsymbol{t}^{*}, v^{*}\right)$, where $\eta^{*}(s)=$ $t_{i}^{*}-t_{i-1}^{*}, \quad s \in[i-1, i), i=1,2, \ldots, 2 l+1$, and $\hat{v}^{*}(s)=v\left(t^{*}(s)\right), \quad s \in[0$, $2 l+1]$.

Proof The proof is similar to the proof for the Theorem 3.2.

Note that the recharge points are fixed. But, there may exist collapses of the switching times. Thus, the exact optimal number of recharge points is equal to $l$ minus the number of recharge points corresponding to the occurrance of redundancies.

\section{FREE RECHARGE LOCATIONS}

With the notation defined in Section 5, we consider the situation in which the recharge locations for a travelling vehicle are free to be optimized. The problem can be formulated as the following optimization problem:

Given the dynamical system (5.2) and (5.3), find a $(t, v, l) \in \mathcal{T} \times$ $\mathcal{V} \times \mathbb{N}$ such that the cost function

$$
g_{4}(\boldsymbol{t}, v, l)=t_{\text {final }}(\boldsymbol{t}, v, l)
$$

is minimized subject to the constraints

$$
q_{\min } \leq q(t) \leq q_{\max }
$$




$$
\begin{aligned}
& 0<p=m\left(a v+b v^{2}+g \sin \theta\right)<P, \\
& 0<t_{i}-t_{i-1}, \quad i=1, \ldots, 2 l+1, \\
& \int_{0}^{t_{\text {final }}} v \mathrm{~d} t=S, \\
& \int_{0}^{t_{i_{j}}} v(t) \mathrm{d} t=\gamma_{i}, \quad j=1, \ldots, m, t_{i_{j}} \in \boldsymbol{t}, \\
& l \leq L,
\end{aligned}
$$

where $S$ is the total distance travelled, $L$ is the the upper bound of the number of the recharge points, $n$ and $l$ are, respectively, the number of battery cells and the number of recharge points, $v$ is the speed, and $\boldsymbol{\gamma}=\left\{\gamma_{1}, \ldots, \gamma_{m}\right\}$ is the vector of the turning points of the undulated road. Let this problem be referred to as Problem $P_{4}$.

\subsection{Transformation}

To solve Problem $P_{4}$, we need to determine the optimal number $l$ of recharge points, the optimal time points $t=\left(t_{1}, \ldots, t_{2 l}\right)$, and the optimal speed $v$. Let us initially choose the optimal number of recharge points to be $k \geq m$. These recharge points include the turning points $\boldsymbol{\gamma}=\left(\gamma_{1}, \ldots, \gamma_{m}\right)$. Then, only the optimal switching time points with a fixed number of recharge points, and the corresponding optimal speed are to be determined optimally. This simplified problem is referred to as Problem $P_{4}^{k}$.

We now apply the CPET [4,5] to Problem $P_{4}^{k}$. Let $s \in[0,2 k+1]$ be a new time variable, and define

$$
\eta(s)=\sum_{i=1}^{2 k+1} \eta_{i} \chi_{[i-1, i)}(s)
$$

where $\chi_{[i-1, i)}(s)$ is the characteristic function on the interval $[i-1, i)$, and the $\eta_{i}^{\prime} s$ are nonnegative constants. Clearly, $\eta(s)$ is a nonnegative piecewise constant function defined on $[0,2 k+1]$ with fixed switching 
times $\{1,2, \ldots, 2 k\}$. The CPET:

$$
\frac{\mathrm{d} t}{\mathrm{~d} s}=\eta(s), \quad t(0)=0
$$

maps $t \in\left[0, t_{\text {final }}\right]$ into $s \in[0,2 k+1]$, where

$$
\eta(s)=t_{i}-t_{i-1}, \quad s \in[i-1, i), \quad i=1,2, \ldots, 2 k+1,
$$

satisfying

$$
\int_{0}^{2 k+1} \eta(s) \mathrm{d} s=t_{\text {final }} .
$$

Let $\Theta$ denote the class of all such enhancing controls. Under the CPET, the system dynamics (5.2) and (5.3) (with $l=k$ ) becomes

$$
\begin{aligned}
& \frac{\mathrm{d}}{\mathrm{d} s}\left(\begin{array}{c}
\hat{q}(s) \\
t(s)
\end{array}\right) \\
& =\left(\begin{array}{ll}
-c_{1} \frac{\left(b_{1} \hat{v}+b_{2} \hat{v}^{2}+g \sin \theta\right) \cdot m}{n}, & s \in[2 i-2,2 i-1), \\
\frac{c_{2}}{\hat{q}+c_{3}}-\exp \left\{c_{4}-c_{5}(t-2 i+1)\right\}, & s \in[2 i-1,2 i), \\
-c_{1} \frac{\left(b_{1} \hat{v}+b_{2} \hat{v}^{2}+g \sin \theta\right) \cdot m}{n}, & s \in[2 k, 2 k+1] \\
\eta(s) &
\end{array}\right)
\end{aligned}
$$

$i=1, \ldots, k$ with the initial condition

$$
\left(\begin{array}{c}
\hat{q}(0) \\
t(0)
\end{array}\right)=\left(\begin{array}{c}
q_{0} \\
0
\end{array}\right)
$$

where $\hat{q}(s)=q(t(s)), \hat{v}(s)=v(t(s)), s \in[0,2 k+1]$, and $i=1, \ldots, k$.

The constraints (6.2) and (6.7) reduce to

$$
\begin{aligned}
& q_{\min } \leq \hat{q}(2 i-1), \quad i=1, \ldots, k+1, \\
& \hat{q}(2 i) \leq q_{\max }, \quad i=1, \ldots, k, \\
& 0<p=m\left(b_{1} \hat{v}+b_{2} \hat{v}^{2}+g \sin \theta\right)<P,
\end{aligned}
$$




$$
\begin{array}{ll}
0<\eta_{i}, & i=1, \ldots, 2 k+1, \\
\int_{0}^{2 k+1} \hat{v} \mathrm{~d} s=S, & \\
\int_{0}^{i_{j}} v(s) \mathrm{d} t=\gamma_{i}, \quad j=1, \ldots, m, \quad i_{j} \in\{1, \ldots, 2 k-1\} .
\end{array}
$$

Let $\mathcal{V}_{4}$ be the set of all those $\hat{v}(s)$ such that the conditions (6.10)(6.15) are satisfied. Problem $P_{4}^{k}$ is now transformed into the following optimal control problem:

Given the dynamical system (6.8) and (6.9), find a $(\eta, \hat{v}) \in \Theta \times \mathcal{V}_{4}$ such that the cost function

$$
\hat{g}_{4}(\boldsymbol{\eta}, \hat{v})=\sum_{i=1}^{2 k+1} \eta_{i}
$$

is minimized subject to the constraints (6.11)-(6.15).

This problem is referred to as Problem $P_{4}^{k 0}$.

Theorem 6.1 Problem $\left(P_{4}^{k 0}\right)$ is equivalent to Problem $\left(P_{4}^{k}\right)$ in the sense that $\left(\eta^{*}, \hat{v}^{*}\right)$ is a solution of Problem $\left(P_{4}^{k 0}\right)$ if and only if $\left(t^{*}, v^{*}\right)$ is a solution of Problem $\left(P_{4}^{k}\right)$, and $\hat{g}_{4}\left(\eta^{*}, \hat{v}^{*}\right)=g_{4}\left(\boldsymbol{t}^{*}, v^{*}\right)$, where $\boldsymbol{\eta}^{*}(\boldsymbol{s})=$ $t_{i}^{*}-t_{i-1}^{*}, \quad s \in[i-1, i), i=1,2, \ldots, 2 k+1$, and $\hat{v}^{*}(s)=v\left(t^{*}(s)\right), s \in[0$, $2 k+1]$.

Proof The proof is similar to the proof for the Theorem 3.2.

The recharge points, besides the $m$ turning points, can be located in any intervals of the undulated road. Let $\boldsymbol{t}=\left(t_{1}, \ldots, t_{2 k}\right)$ be optimal solution of Problem $\left(P_{4}^{k}\right)$ for a fixed positive integer $k$, and fixed permutation of the recharge points in the intervals of the undulated road. We propose the following algorithm for finding the optimal number of recharge points:

\section{Algorithm B}

1. Choose an initial guess $k=k_{0}$.

2. Let $t_{0}=0, t_{2 k+1}=t_{\text {final }}$. Solve Problem $\left(P_{4}^{k}\right)$ to obtain $\boldsymbol{t}=\left(t_{1}, \ldots, t_{2 k}\right)$. Let $\hat{g}_{4}^{k}$ be the optimal cost. 
3. If $k=L$, stop. Find $k^{*}$, which denotes a permutation of $k$ recharge points in the intervals of the undulated road, such that $\hat{g}_{4}^{k^{*}} \leq \hat{g}_{4}^{l}$ for all $k=k_{0}, \ldots, L$, and all permutations of the $k$ recharge points in the intervals of the undulated road. $k^{*}$ is the optimal solution of the recharge points. Otherwise, let $k=k+1$ and go to step 2 .

\section{NUMERICAL EXPERIMENTS}

We now consider some numerical examples of the problems formulated in Sections 3-6. All the examples below were solved using MISER3.2 [2,3] in Fortran double precision on a Unix Workstation.

In Examples 1 and 2, the road connecting the two cities is assumed to be even.

Example 1 Choose $S=600, P=50, m_{1}=1400, L=5$, and $n=80$ in Problem $\left(P_{1}\right)$. The dynamical system is

$$
\begin{aligned}
& \frac{\mathrm{d} q}{\mathrm{~d} t} \\
& = \begin{cases}-\frac{1}{100} \frac{\left(v / 40+v^{2} / 1200\right) \cdot 1400}{80}, & t \in\left[t_{2 i-2}, t_{2 i-1}\right), \\
\frac{2000}{q+50}-\exp \left\{2.5-3.0\left(t_{2 i}-t_{2 i-1}\right)\left(t-t_{2 i-1}\right)\right\}, & t \in\left[t_{2 i-1}, t_{2 i}\right), \\
-\frac{1}{100} \frac{\left(v / 40+v^{2} / 1200\right) \cdot 1400}{80}, & t \in\left[t_{2 l}, t_{2 l+1}\right], \\
i=1, \ldots, l & \end{cases}
\end{aligned}
$$

with initial condition

$$
q(0)=60
$$

Then, Problem $P_{1}$ becomes:

Given the dynamical system (7.1) and (7.2), find $\boldsymbol{t}=\left\{t_{1}, t_{2}, \ldots, t_{2 l}\right\}$, $v, l$, such that

$$
g_{1}(\boldsymbol{t}, v, l)=t_{\text {final }}(\boldsymbol{t}, v, l)
$$


is minimized subject to the constraints

$$
\begin{aligned}
& 20 \leq q(t) \leq 80, \\
& 0<p<50, \\
& 0<t_{i}-t_{i-1}, \quad i=1, \ldots, 2 l+1, \\
& \int_{0}^{t_{\text {final }}} v \mathrm{~d} t=600, \\
& \quad l \leq 5
\end{aligned}
$$

We choose $k_{0}=2$ in Algorithm A. Consider the case in which $k=4$.

Under the CPET given in Section 3, we have:

Given the dynamical system

$$
\begin{aligned}
& \frac{\mathrm{d} \hat{q}}{\mathrm{~d} s} \\
& = \begin{cases}-\frac{1}{100} \eta \frac{\left(\hat{v} / 40+\hat{v}^{2} / 1200\right) \cdot 1400}{80}, & s \in[2 i-2,2 i-1), \\
\eta \frac{2000}{q+50}-\exp \left\{2.5-3.0 \eta_{2 i}(s-2 i+1)\right\}, & s \in[2 i-1,2 i), \\
-\frac{1}{100} \eta \frac{\left(\hat{v} / 40+\hat{v}^{2} / 1200\right) \cdot 1400}{80}, & s \in[8,9] \\
i=1, \ldots, 4, & \end{cases}
\end{aligned}
$$

with initial condition

$$
\hat{q}(0)=60,
$$

find parameter vectors $\eta$ and $\hat{v}$ such that

$$
\hat{g}_{1}(\boldsymbol{\eta}, \hat{v})=\sum_{i=1}^{9} \eta_{i}
$$

is minimized subject to

$$
\begin{array}{ll}
20 \leq \hat{q}(2 i-1) & i=1, \ldots, 5 \\
\hat{q}(2 i) \leq 80 & i=1, \ldots, 4 \\
0<p \leq 50, & \\
0<\eta_{i} & i=1, \ldots, 9 \\
\int_{0}^{9} \hat{v} \mathrm{~d} s=600 . &
\end{array}
$$


TABLE I The optimal switching times and recharge points for Example 1

\begin{tabular}{cccccc}
\hline$i$ & 1 & 2 & 3 & 4 & 5 \\
\hline$t_{i}$ & 3.2000 & 5.4852 & 9.1523 & 11.094 & 14.275 \\
$x_{i}$ & 191.08 & 191.08 & 410.06 & 410.06 & 600 \\
\hline
\end{tabular}

The optimal switching times and recharge points are listed in Table I, in which the optimal switching times are $3.200,5.485,9.153$, and 11.09, while the optimal recharge points are 191.08 and 410.06 . The corresponding optimal speed is 59.71 with minimum travelling time 14.275.

Figure 1 contains the optimal recharge plan, the optimal switching times and the optimal driving speed.

Example 2 In Problem $P_{2}$, choose $S=600, P=50, m=1470$ and $n=87$. The recharge locations are restricted at 130, 240,300, 420, and 490. The dynamical system is:

$$
\begin{aligned}
& \frac{\mathrm{d} q}{\mathrm{~d} t} \\
& = \begin{cases}-\frac{1}{100} \frac{\left(v / 40+v^{2} / 1200\right) \cdot 1470}{87}, & t \in\left[t_{2 i-2}, t_{2 i-1}\right), \\
\frac{2000}{q+50}-\exp \left\{2.5-3.0\left(t_{2 i}-t_{2 i-1}\right)\left(t-t_{2 i-1}\right)\right\}, & t \in\left[t_{2 i-1}, t_{2 i}\right), \\
-\frac{1}{100} \frac{\left(v / 40+v^{2} / 1200\right) \cdot 1470}{87}, & t \in\left[t_{10}, t_{11}\right], \\
i=1, \ldots, 5 & \end{cases}
\end{aligned}
$$

with initial condition

$$
q(0)=60
$$

Problem $\left(P_{2}\right)$ becomes:

Given the dynamical system (7.3) and (7.4), find $\boldsymbol{t}=\left\{t_{1}, t_{2}, \ldots, t_{11}\right\}$ and $v$ such that

$$
g_{2}(\boldsymbol{t}, v)=t_{\text {final }}(\boldsymbol{t}, v)
$$



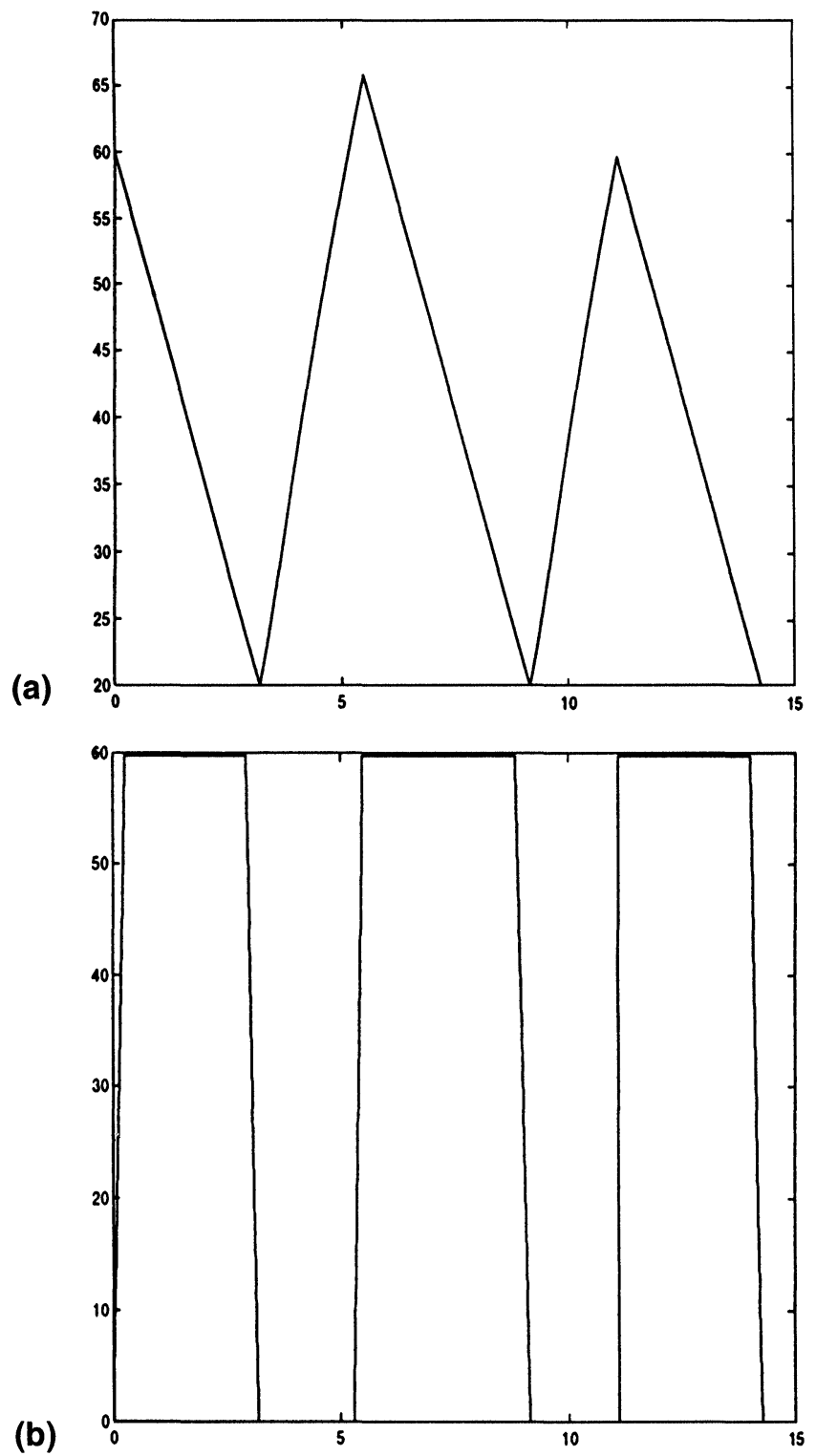

FIGURE 1 The optimal driving strategy for Example 1. (a) optimal recharge plan (b) optimal driving speed. 
is minimized subject to the constraints

$$
\begin{aligned}
& 20 \leq q(t) \leq 80, \\
& 0<p<50, \\
& 0<t_{i}-t_{i-1}, \quad i=1, \ldots, 11, \\
& \int_{0}^{t_{11}} v \mathrm{~d} t=600, \\
& \int_{0}^{t_{2 i-1}} v \mathrm{~d} t=\tau_{i}, \quad i=1,2, \ldots, 5,
\end{aligned}
$$

where $\left(\tau_{1}, \ldots, \tau_{5}\right)=(130,240,300,420,490)$. Under the CPET transform, we have:

Given the dynamics

$$
\begin{aligned}
& \frac{\mathrm{d} \hat{q}}{\mathrm{~d} s} \\
& = \begin{cases}-\frac{1}{100} \eta \frac{\left(\hat{v} / 40+\hat{v}^{2} / 1200\right) \cdot 1470}{87}, & s \in[2 i-2,2 i-1), \\
\eta \frac{2000}{q+50}-\exp \left\{2.5-3.0 \eta_{2 i}(s-2 i-1)\right\}, & s \in[2 i-1,2 i), \\
-\frac{1}{100} \eta \frac{\left(\hat{v} / 40+\hat{v}^{2} / 1200\right) \cdot 1470}{87}, & s \in[10,11], \\
i=1, \ldots, 5 & \end{cases}
\end{aligned}
$$

with initial condition

$$
\hat{q}(0)=60,
$$

find parameter vectors $\eta$ and $\hat{v}$ such that

$$
\hat{g}_{2}(\boldsymbol{\eta}, \hat{v})=\sum_{i=1}^{11} \eta_{i}
$$

is minimized subject to

$$
\begin{array}{ll}
20 \leq \hat{q}(2 i-1), & i=1, \ldots, 6 \\
\hat{q}(2 i) \leq 80, & i=1, \ldots, 5, \\
0<p=\leq 50, & \\
0<\eta_{i}, & i=1, \ldots, 11
\end{array}
$$




$$
\begin{aligned}
& \int_{0}^{11} \hat{v} \mathrm{~d} s=600 \\
& \int_{0}^{2 i-1} \hat{v}(s) \mathrm{d} s=\tau_{i} \quad i=1,2, \ldots, 5 .
\end{aligned}
$$

The optimal switching times and recharge points, listed in Table II, show that there are four redundancies $t_{3}=t_{4}, t_{7}=t_{8}$. The lengths of the recharge intervals $\left[t_{3}, t_{4}\right]$, and $\left[t_{7}, t_{8}\right]$ are all equal to zero. Thus, the actual recharge intervals are $\left[t_{1}, t_{2}\right],\left[t_{5}, t_{6}\right]$ and $\left[t_{9}, t_{10}\right]$. The optimal switching times are $2.242,3.330,6.262,8.089,11.36$, and 12.39 , while the optimal recharge points are 130,300 , and 488 . The corresponding optimal speed is 57.76 with minimum travelling times 14.292.

Figure 2 contains the optimal recharge plan, the optimal switching times and the optimal driving speed.

In Examples 3 and 4, the road connecting the two cities is assumed to be undulated with two tuning points located at 350 and 550 , respectively. Therefore $\boldsymbol{\gamma}=(350,550)$. The angles of slope of the road on the intervals $(0,350),(350,550)$, and $(550,600)$ are $4.4^{\circ}, 174^{\circ}$, and $5.86^{\circ}$, respectively.

Example 3 In Problem $\left(P_{3}\right)$, choose $P=50, m=1440$, and $n=84$. The recharge locations are restricted at 150, 250, 350, and 470. All turning points of the undulated road are included in the recharge points. Thus, the recharge points are $150,250,350,470$, and 550 . The dynamic system is

$$
\begin{aligned}
& \frac{\mathrm{d} q}{\mathrm{~d} t} \\
& = \begin{cases}-\frac{1}{100} \frac{\left(v / 40+v^{2} / 1200+g \sin \theta\right) \cdot 1380}{78}, & t \in\left[t_{2 i-2}, t_{2 i-1}\right), \\
\frac{2000}{q+50}-\exp \left\{2.5-3.0\left(t_{2 i}-t_{2 i-1}\right)\left(t-t_{2 i-1}\right)\right\}, & t \in\left[t_{2 i-1}, t_{2 i}\right) \\
-\frac{1}{100} \frac{\left(v / 40+v^{2} / 1200+g \sin \theta\right) \cdot 1380}{78}, & t \in\left[t_{8}, t_{10}\right] \\
i=1, \ldots, 4 & \end{cases}
\end{aligned}
$$

TABLE II The optimal switching times and recharge points for Example 2

\begin{tabular}{cccccccccccc}
\hline$i$ & 1 & 2 & 3 & 4 & 5 & 6 & 7 & 8 & 9 & 10 & 11 \\
\hline$t_{i}$ & 2.24 & 3.33 & 5.23 & 5.23 & 6.26 & 8.09 & 10.16 & 10.16 & 11.37 & 12.39 & 14.29 \\
$x_{i}$ & 130.0 & 130.0 & 240.0 & 240.0 & 300.0 & 300.0 & 418.7 & 418.7 & 488.4 & 488.4 & 600.0 \\
\hline
\end{tabular}



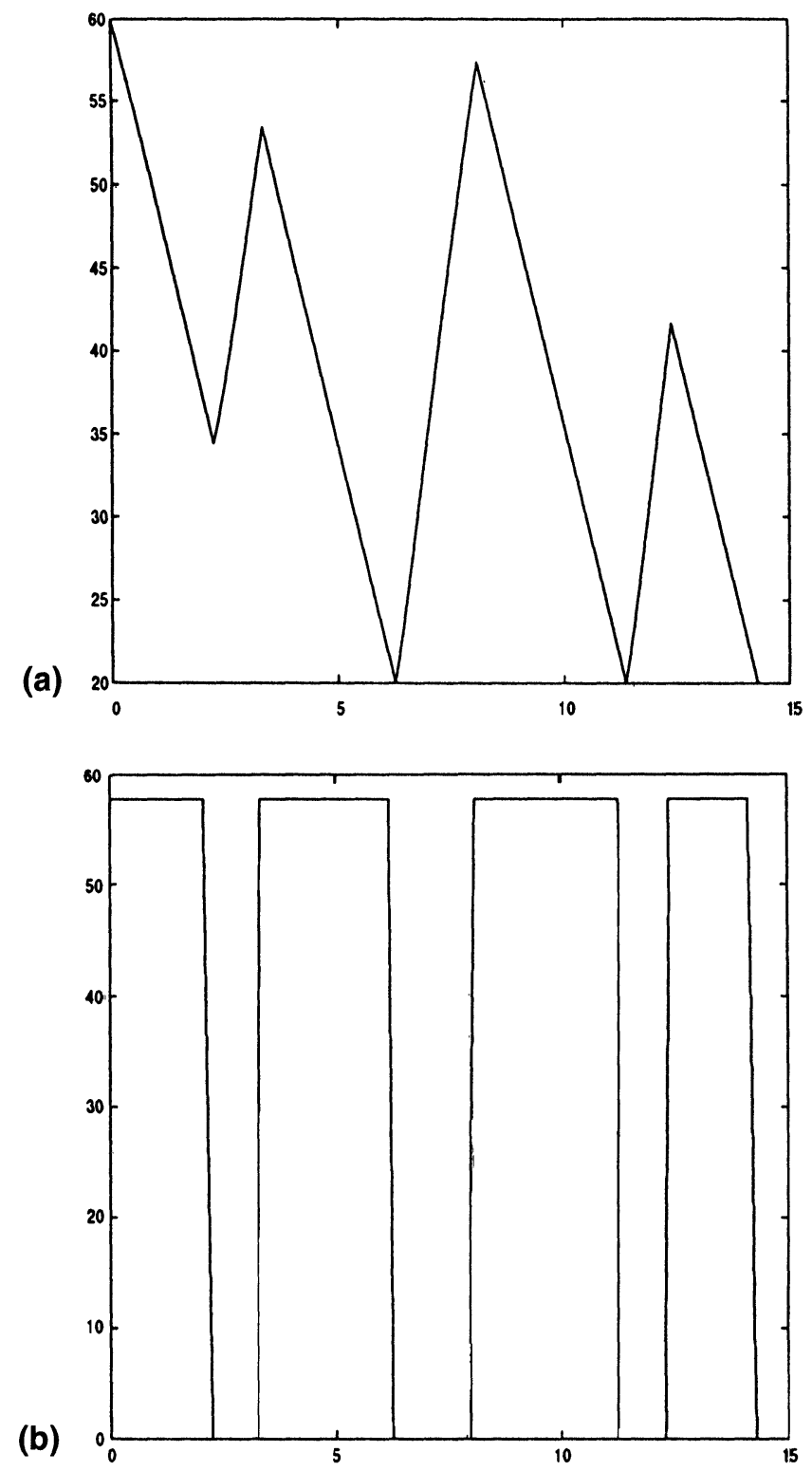

FIGURE 2 The optimal driving strategy for Example 2. (a) Optimal recharge plan, (b) optimal driving speed. 
with initial condition

$$
q(0)=60
$$

Problem $\left(P_{3}\right)$ then becomes:

Given the dynamical system (7.5) and (7.6), find $\boldsymbol{t}=\left\{t_{1}, t_{2}, \ldots, t_{9}\right\}$, and $v$ such that

$$
g_{3}(t, v)=t_{\text {final }}(t, v)
$$

is minimized subject to the constraints

$$
\begin{aligned}
& 20 \leq q(t) \leq 80, \\
& 0<p<50, \\
& 0<t_{i}-t_{i-1}, \quad i=1, \ldots, 10, \\
& \int_{0}^{t_{10}} v \mathrm{~d} t=600, \\
& \int_{0}^{t_{2 i-1}} v \mathrm{~d} t=\tau_{i}, \quad i=1, \ldots, 5,
\end{aligned}
$$

where $\left(\tau_{1}, \ldots, \tau_{5}\right)=(150,250,350,470,550)$. Under the CPET given in Section 5, we have:

Given the dynamics

$$
\begin{aligned}
& \frac{\mathrm{d} \hat{q}}{\mathrm{~d} s} \\
& \quad= \begin{cases}-\frac{1}{100} \eta \frac{\left(\hat{v} / 40+\hat{v}^{2} / 1200+g \sin \theta\right) \cdot 1380}{78}, & s \in[2 i-2,2 i-1), \\
\eta \frac{2000}{q+50}-\exp \left\{2.5-3.0 \eta_{2 i}(s-2 i+1)\right\}, & s \in[2 i-1,2 i), \\
-\frac{1}{100} \eta \frac{\left(\hat{v} / 40+\hat{v}^{2} / 1200+g \sin \theta\right) \cdot 1380}{78}, & s \in[8,10], \\
i=1, \ldots, 4 & \end{cases}
\end{aligned}
$$


with initial condition

$$
\hat{q}(0)=60
$$

find parameter vectors $\eta$ and $\hat{v}$ such that

$$
\hat{g}_{3}(\eta, \hat{v})=\sum_{i=1}^{10} \eta_{i}
$$

is minimized subject to

$$
\begin{array}{ll}
20 \leq \hat{q}(i), & i=1,3,5,7,9,10, \\
\hat{q}(2 i) \leq 80, & i=1, \ldots, 4, \\
0<p \leq 50, & i=1, \ldots, 10 \\
0<\eta_{i}, & \\
\int_{0}^{10} \hat{v} \mathrm{~d} s=600, & \\
\int_{0}^{2 i-1} \hat{v}(s) \mathrm{d} s=\tau_{i}, & i=1, \ldots, 5 .
\end{array}
$$

The optimal switching times and recharge points, listed in Table III, show that there exist redundancies: $t_{3}=t_{4}, t_{7}=t_{8}$. The lengths of the recharge intervals $\left[t_{3}, t_{4}\right]$ and $\left[t_{7}, t_{8}\right]$ are all equal to zero. The actual recharge intervals are $\left[t_{1}, t_{2}\right]$ and $\left[t_{5}, t_{6}\right]$. Therefore, the optimal recharge locations are 150 , and 350 . The optimal switching times are $2.778,5.010,8.714,11.256$, and 14.204. The corresponding optimal speeds are 54.0,67.8, and 51.8. The minimum travelling time is 15.170 .

Figure 3 contains the optimal recharge plan, the optimal switching times, and the optimal driving speed.

TABLE III The optimal switching times and recharge points for Example 3

\begin{tabular}{ccccccccccc}
\hline$i$ & 1 & 2 & 3 & 4 & 5 & 6 & 7 & 8 & 9 & 10 \\
\hline$t_{i}$ & 2.778 & 5.010 & 6.862 & 6.862 & 8.714 & 11.26 & 13.02 & 13.02 & 14.20 & 15.17 \\
$x_{i}$ & 150.0 & 150.0 & 250.0 & 250.0 & 350.0 & 350.0 & 470.1 & 470.1 & 550.1 & 600 \\
\hline
\end{tabular}



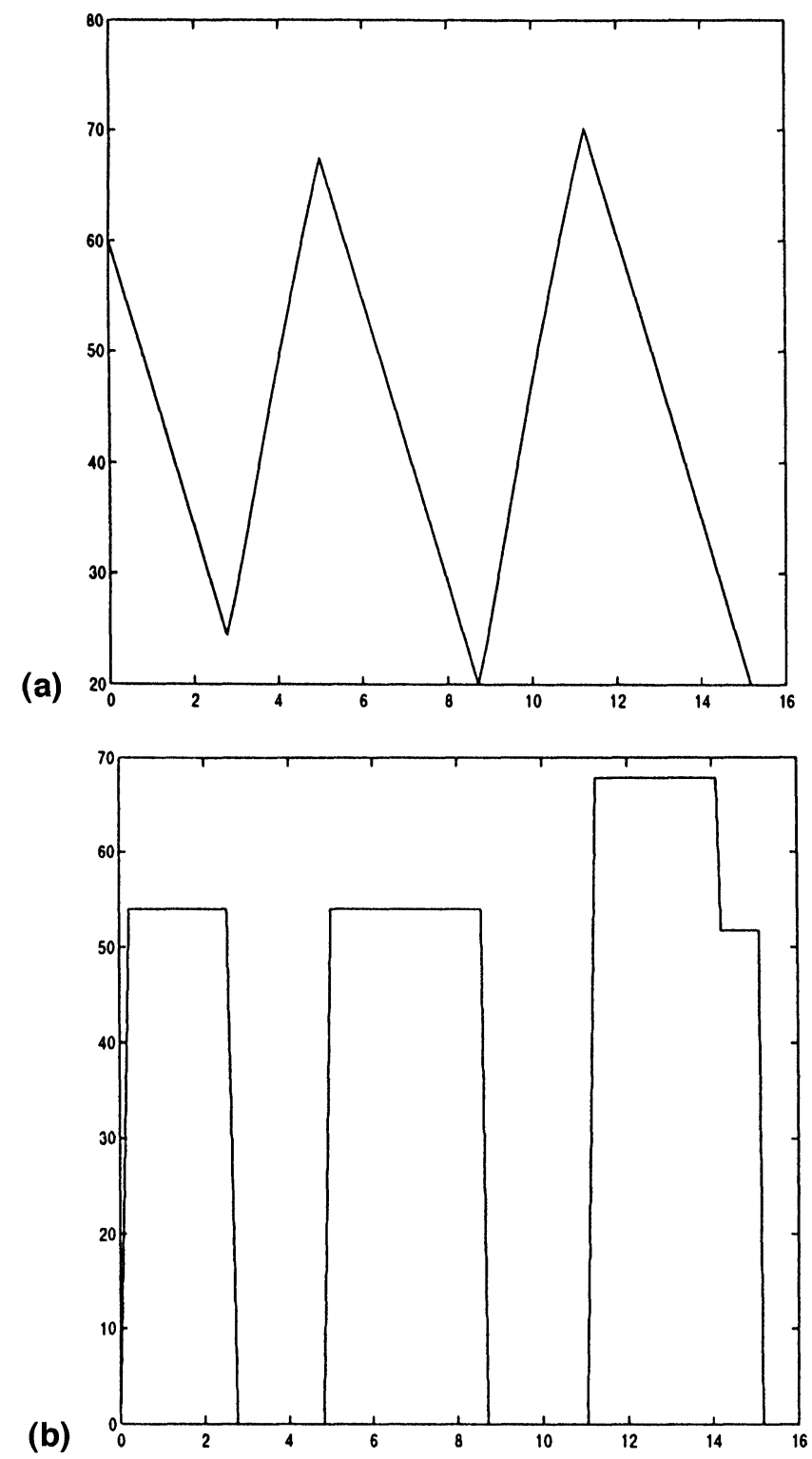

FIGURE 3 The optimal driving strategy for Example 3. (a) Optimal recharge plan, (b) optimal driving speed. 
Example 4 In Problem $\left(P_{4}\right)$, choose $S=600, P=50, m_{1}=1440$, $L=5$, and $n=84$. The dynamical system is:

$$
\begin{aligned}
& \frac{\mathrm{d} q}{\mathrm{~d} t} \\
& = \begin{cases}-\frac{1}{100} \frac{\left(v / 40+v^{2} / 1200\right) \cdot 1440}{84}, & t \in\left[t_{2 i-2}, t_{2 i-1}\right), \\
\frac{2000}{q+50}-\exp \left\{2.5-3.0\left(t_{2 i}-t_{2 i-1}\right)\left(t-t_{2 i-1}\right)\right\}, & t \in\left[t_{2 i-1}, t_{2 i}\right), \\
-\frac{1}{100} \frac{\left(v / 40+v^{2} / 1200\right) \cdot 1440}{84}, & t \in\left[t_{2 l}, t_{2 l+1}\right], \\
i=1, \ldots, l & \end{cases}
\end{aligned}
$$

with initial condition

$$
q(0)=60
$$

Problem $\left(P_{4}\right)$ becomes:

Given the dynamical system (7.7) and (7.8), find $\boldsymbol{t}=\left\{t_{1}, t_{2}, \ldots, t_{2 l}\right\}$, $v, l$, such that

$$
g_{4}(\boldsymbol{t}, v, l)=t_{\text {final }}(\boldsymbol{t}, v, l)
$$

is minimized subject to the constraints

$$
\begin{aligned}
& 20 \leq q(t) \leq 80, \\
& 0<p<50, \\
& 0<t_{i}-t_{i-1}, \quad i=1, \ldots, 2 l+1, \\
& \int_{0}^{t_{\text {final }}} v \mathrm{~d} t=600, \\
& \int_{0}^{t_{i_{j}}} v(t) \mathrm{d} t=\gamma_{j}, \quad j=1,2, \quad t_{i_{j}} \in \boldsymbol{t}, \\
& l \leq 10
\end{aligned}
$$

where $\boldsymbol{\gamma}=(350,550)$.

We choose $k_{0}=3$ in Algorithm B. Consider the case in which $k=5$. 
Under the CPET given in Section 6, the problem becomes:

Given the dynamical system

$$
\frac{\mathrm{d} \hat{q}}{\mathrm{~d} s}= \begin{cases}-\frac{1}{100} \eta \frac{\left(\hat{v} / 40+\hat{v}^{2} / 1200\right) \cdot 1440}{84}, & s \in[2 i-2,2 i-1), \\ \eta \frac{2000}{q+50}-\exp \left\{2.5-3.0 \eta_{2 i}(s-2 i+1)\right\}, & s \in[2 i-1,2 i), \\ -\frac{1}{100} \eta \frac{\left(\hat{v} / 40+\hat{v}^{2} / 1200\right) \cdot 1440}{84}, & s \in[10,11], \\ i=1, \ldots, 5 & \end{cases}
$$

with initial condition

$$
\hat{q}(0)=60,
$$

find parameter vectors $\eta$ and $\hat{v}$ such that

$$
\hat{g}_{4}(\eta, \hat{v})=\sum_{i=1}^{11} \eta_{i}
$$

is minimized subject to

$$
\begin{array}{ll}
20 \leq \hat{q}(2 i-1), & i=1, \ldots, 6, \\
\hat{q}(2 i) \leq 80, & i=1, \ldots, 5, \\
0<p \leq 50, & i=1, \ldots, 11, \\
0<\eta_{i}, & \\
\int_{0}^{i_{j}} v(t) \mathrm{d} t=\gamma_{j}, & j=1,2, \quad i_{j} \in\{1,3,5,7,9\}, \\
\int_{0}^{11} \hat{v} \mathrm{~d} s=600 . &
\end{array}
$$

The optimal switching times and recharge points are listed in Table IV. The optimal switching times are 3.360, 5.294, 8.623, 11.02,

TABLE IV The optimal switching times and recharge points for Example 4

\begin{tabular}{lcccccc}
\hline$i$ & 1 & 2 & 3 & 4 & 5 & 6 \\
\hline$t_{i}$ & 3.3600 & 5.2944 & 8.6227 & 11.018 & 14.028 & 15.026 \\
$x_{i}$ & 175.83 & 175.83 & 350.00 & 350.00 & 550.00 & 600 \\
\hline
\end{tabular}



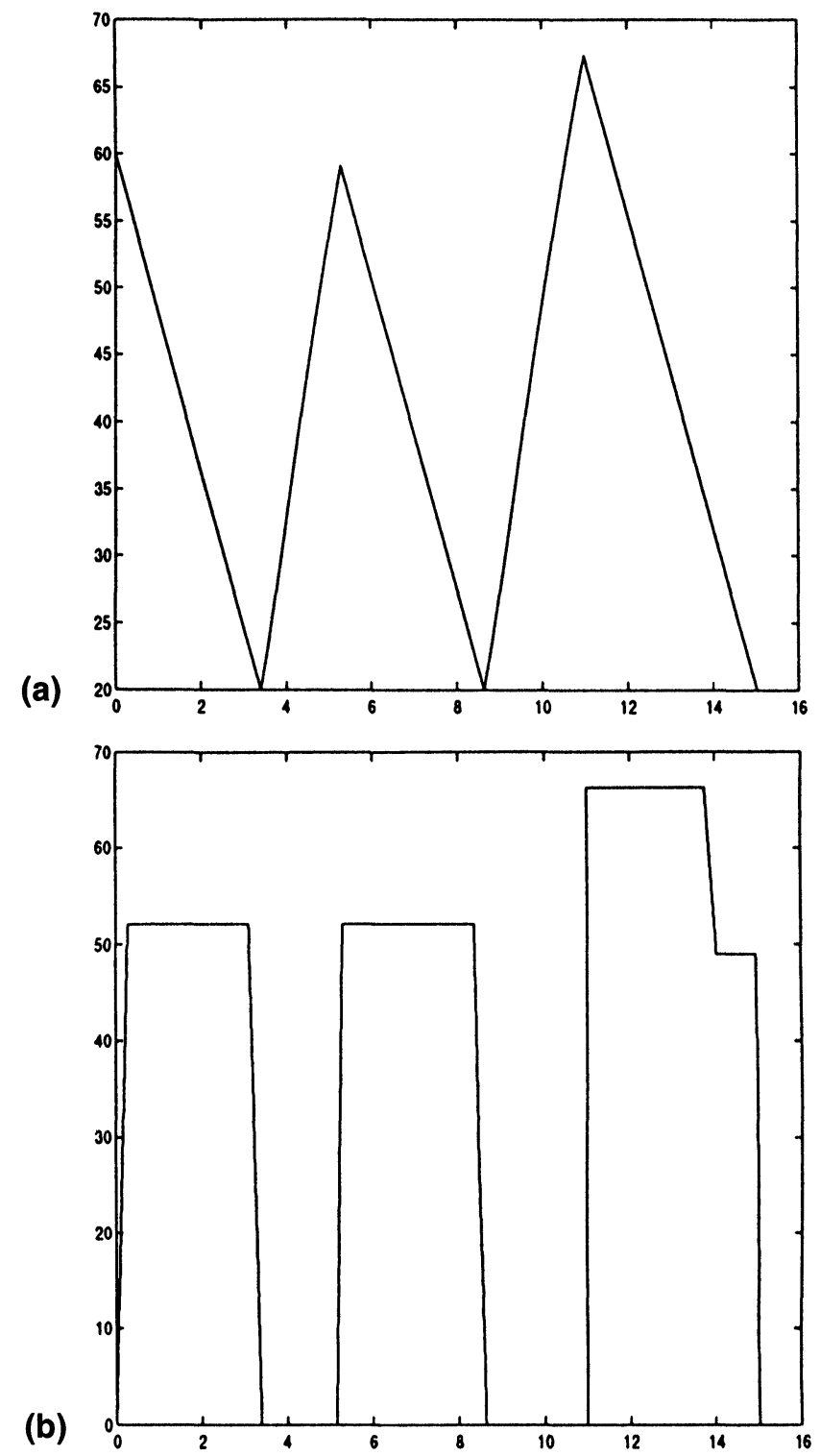

FIGURE 4 The Optimal Driving Strategy for Example 4. (a) Optimal recharge plan, (b) optimal driving speed. 
and 14.03, the optimal recharge locations are 175.8, and 350.0. The corresponding optimal speeds are $52.06,66.22$, and 49.79 , respectively. The minimum travelling time is $\mathbf{1 5 . 0 2 6 .}$

Figure 4 contains the optimal recharge plan, the optimal switching times and the optimal driving speed.

\section{References}

[1] D. Bunch et al., "Demand for clean fuelled vehicles in California: a discrete choice stated preference survey", Conference on Transportation and Global Climate Change, Pacific Grove California (1991).

[2] L.S. Jennings, M.E. Fisher, K.L. Teo and C.J. Goh, MISER3.2 Optimal Control Software: Theory and User Manual, EMCOSS Pty Ltd. (1990).

[3] L.S. Jennings, M.E. Fisher, K.L. Teo and C.J. Goh, MISER3 - Version 2.0 (1997) Optimal Control Software: Theory and User Manual, EMCOSS Pty Ltd. (1997).

[4] H.W.J. Lee, K.L. Teo, L.S. Jennings and V. Rehbock, Control parameterization enhancing technique for time optimal control problems, Dynamic Systems and Applications, 6 (1997) 243-262.

[5] H.W.J. Lee, K.L. Teo, V. Rehbock and L.S. Jennings, Control parameterization enhancing technique for optimal discrete-valued control problems, Automatica, to appear.

[6] L. O'Connor, "Energizing the batteries for electric cars," Mechanical Engineering (1993) 73-75.

[7] A. Taylor, "Electric cars, special report." Business Week (1994) 104-114.

[8] G. Tyler, "Motor vehicle are poisoning us", Management Services, Journal of Management Services, Section New Technol., 38(2) (1994) 26-28. 


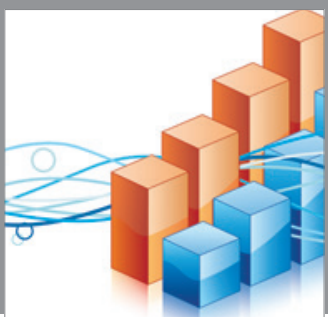

Advances in

Operations Research

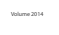

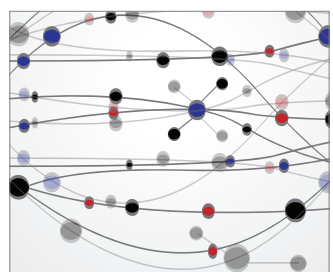

\section{The Scientific} World Journal
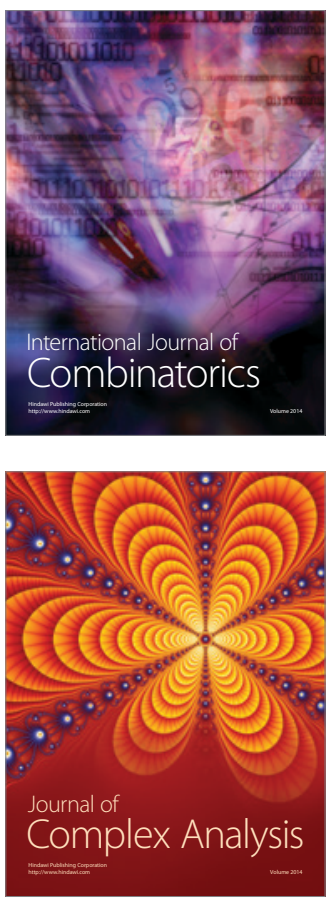

International Journal of

Mathematics and

Mathematical

Sciences
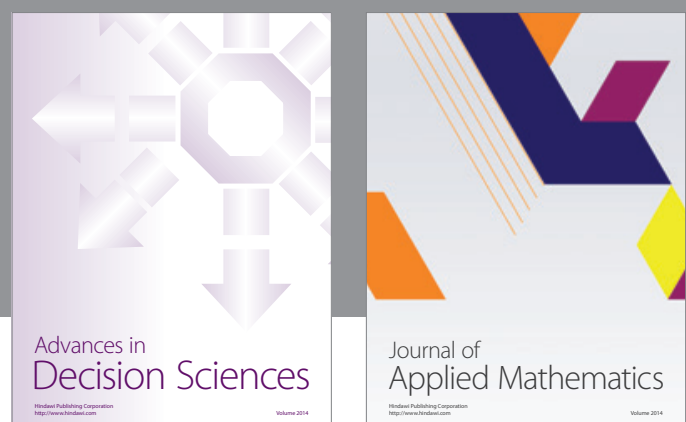

Journal of

Applied Mathematics
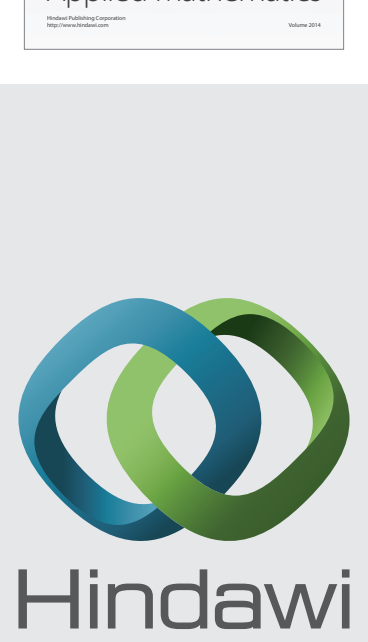

Submit your manuscripts at http://www.hindawi.com
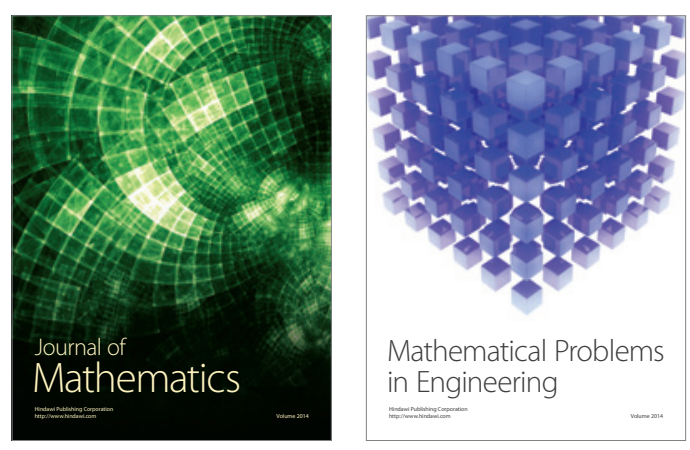

Mathematical Problems in Engineering
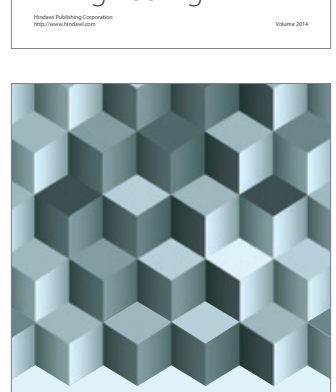

Journal of

Function Spaces
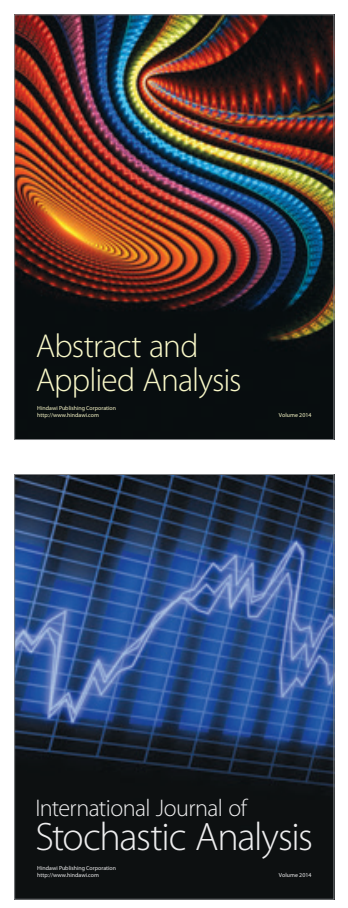

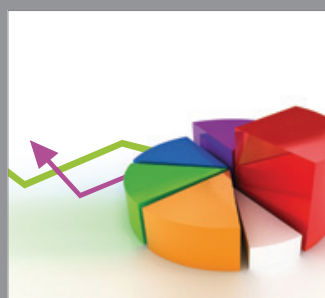

ournal of

Probability and Statistics

Promensencen
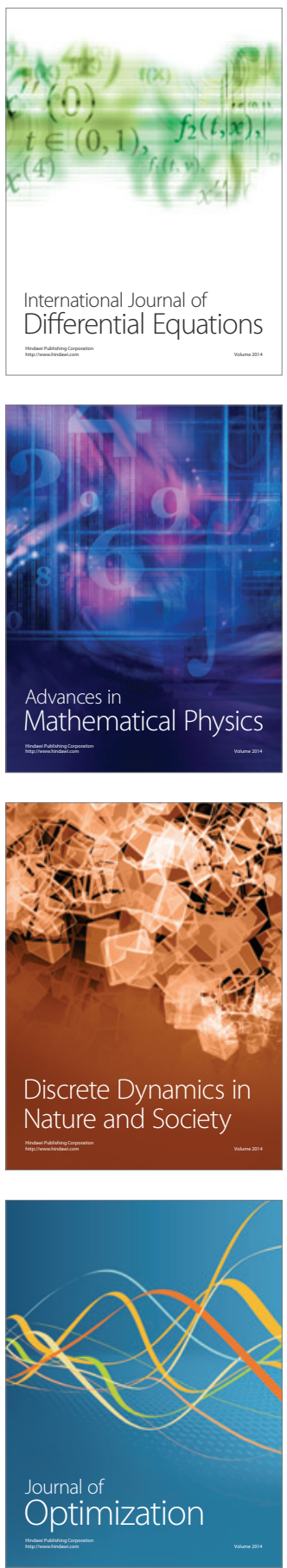